\title{
The Utility of Sulfonylhydrazines in the Study and Potential Treatment of African Sleeping Sickness
}

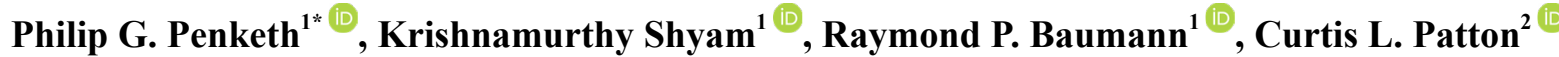 \\ ${ }^{1}$ Department of Pharmacology, Yale University School of Medicine, New Haven, Connecticut, United States \\ ${ }^{2}$ MacArthur Center for Parasitology and Tropical Medicine, Yale University School of Medicine, New Haven, Connecticut, United \\ States \\ E-mail: philip.penketh@gmail.com
}

Received: 19 October 2021; Revised: 23 November 2021; Accepted: 23 November 2021

\begin{abstract}
African sleeping sickness is an invariably fatal disease caused by Trypanosoma brucei group trypanosomes and transmitted by the bite of a Tsetse fly. In the human bloodstream, there are two main forms: long slender rapidly proliferating forms and biochemically distinct, non-proliferating short stumpy forms. These short stumpy forms are preadapted for life in the Tsetse fly gut and cannot sustain or initiate infection in the mammalian host. It was observed that exposure of long slender bloodstream trypomastigotes to a wide range of methylating agents could induce the entire population to differentiate into short stumpy forms. The methylators varied in their nucleophile preference from hard oxygen targets to soft thiol targets. Despite this wide variability, the methylators were all almost equivalently active. It was therefore assumed that the salient target was likely nitrogen of intermediate hardness/softness which would be equivalently targeted by both groups of methylators. A number of sulfonylhydrazine methylators including prodrug forms, with improved biological distribution in animals, were synthesized. In addition, some amidine moiety containing methylators were also synthesized to exploit the propensity of long slender trypanomastigotes to accumulate agents containing this chemical group, in an attempt to enhance their efficacy. These agents may be of utility in the study of differentiation in T. brucei group trypanosomes, and the production of large quantities of short stumpy forms for biochemical studies. Additionally, they could have therapeutic potential in the treatment of late-stage African sleeping sickness.
\end{abstract}

Keywords: African sleeping sickness, Trypanosoma brucei, differentiation, methylating agents, amidine, sulfonylhydrazines

African sleeping sickness is caused by Trypanosoma brucei group trypanosomes, which are the parasitic protozoans. Trypanosoma brucei brucei is a parasite of cattle, while Trypanosoma brucei gambiense and Trypanosoma brucei rhodesiense cause an invariably fatal disease in humans if untreated. The disease is responsible for approximately 50,000 deaths per year in Sub-Saharan Africa [1]. These trypanosomes have a complex life cycle (Figure 1A) and are transmitted by the bite of a Tsetse fly, which injects infective metacyclic forms that differentiate into long slender trypomastigotes, which inhabit the bloodstream and body fluids, and can cross the blood-brain barrier (BBB) [2].

These long slender forms are essentially glycolytic and divide in the bloodstream and tissue fluids [2]. Towards the peak of the parasitemia, a large proportion of the long slender forms differentiate into short stumpy trypomastigotes [2]. This form is terminally differentiated and incapable of division in the mammalian host, and is preadapted for uptake

Copyright (C2021 Philip G. Penketh, et al.

DOI: https://doi.org/10.37256/acbr.1120221217

This is an open-access article distributed under a CC BY license

(Creative Commons Attribution 4.0 International License)

https://creativecommons.org/licenses/by/4.0/ 


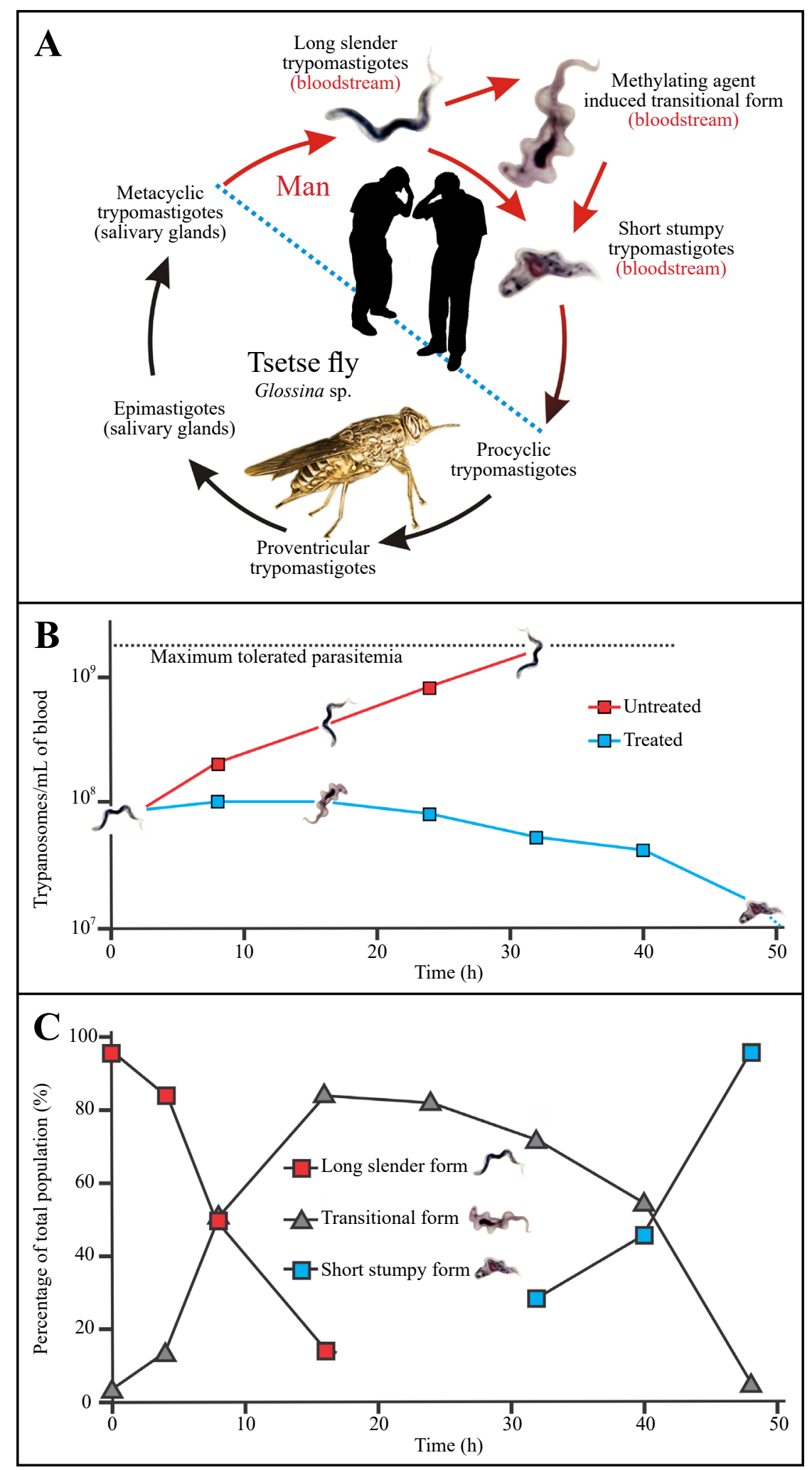

Figure 1. The life cycle of T. b. rhodesiense and the effects of KS90 on parasitemia levels and trypanosome morphology in infected mice: (A) The complex life cycle of $T . b$. rhodesiense involves a proportion of the bloodstream trypanosome population differentiating into specialized short stumpy forms that are preadapted to survival in the Tsetse fly but are incapable of sustaining the infection in the mammalian host; (B) The effects of KS90 (0.02 $\mathrm{mmol} / \mathrm{kg}$ every $8 \mathrm{~h}$ ) on the parasitemia levels. In the absence of treatment, the parasitemia rapidly rises to lethal levels. Treatment with KS 90 quickly halts parasite proliferation and triggers differentiation to short stumpy forms; and (C) The relative proportions of long slender trypomastigote forms, transitional forms, and short stumpy trypomastigotes versus time following the above treatment with KS90. The entire trypanosome population can be induced to differentiate eradicating the infection in mice. 
and replication in the Tsetse fly. Short stumpy forms are less glycolytic and their respiration can be supported by $\alpha$-ketoglutarate $[2,3]$. The vast majority of the short stumpy forms are not taken up by Tsetse flies and die over the course of a day or so. The parasites have a protective coat of variant surface glycoprotein (VSG), and the dying short stumpy forms (and a small proportion of the long slender forms undergoing antigenic variation) shed their VSG coats and immunize the host against this variant [2]. This results in the immune system largely clearing the parasites expressing that particular VSG. However, a small proportion of the slender forms will have changed their VSG to a new unrecognized form and these will initiate the next wave of parasitemia [2]. This process is repeated until the death of the host. The undulating waves of infection delay the death of the host and increase the probability of transmission to a Tsetse fly. In the laboratory, T. b. brucei long slender forms are cultured in mice. Mice appear to present a very weak immune response to many of these rapidly dividing laboratory strains, and infection with a single cell can result in the death of the mouse from a single fulminating wave of parasitemia. In some of these acute strains, a few short stumpy forms begin to appear towards the peak of parasitemia, but in other more highly monomorphic strains, no short stumpy forms are seen even at the highest tolerated parasite burdens [4]. These strains are traditionally used in laboratories because they allow large amounts of parasites to be obtained for experimental purposes.

We initially investigated the actions of KS90, a simple methylating sulfonylhydrazine (Figure 2) on trypanosome infections based on a rather vague premise that like cancer cells $T$. b. brucei could be targeted with alkylating agents based solely on their rapid proliferation rate. We observed that a single dose of KS90 $(0.2 \mathrm{mmol} / \mathrm{kg})$ immediately halted trypanosome proliferation and transformed the cells over 8 to $24 \mathrm{~h}$ into bizarre forms containing multiple nuclei and kinetoplasts. As these abnormal cells disappeared from the bloodstream over 48 to $72 \mathrm{~h}$, it was noticed that the last few remaining trypanosomes to disappear from the bloodstream had acquired short stumpy form morphology. Furthermore, when KS90 was administered at repetitive low doses of $0.02 \mathrm{mmol} / \mathrm{kg}$ every $8 \mathrm{~h}$ for a total of $48 \mathrm{~h}$, it induced the entire population of trypanosomes to differentiate into the biochemically distinct short stumpy forms in a synchronous manner even in the case of extremely monomorphic strains (Figure 1B and 1C) [3]. Mice infected with $10^{6}$ trypanosomes normally die with a parasite burden of $\sim 1-2 \times 10^{9}$ trypanosomes $/ \mathrm{mL}$ of blood after $\sim 4$ days. However, if treated using either a single high dose or repetitive low dose regimens at $\sim 3$ days post-infection, even when the parastemias were as high as $5 \times 10^{8}$ cells $/ \mathrm{mL}$ of blood, cures were still possible. The longer-lived KS90 prodrug KS90T (Figure 2) was more efficacious than KS90 itself, producing $100 \%$ cures at well-tolerated doses [5, 6].

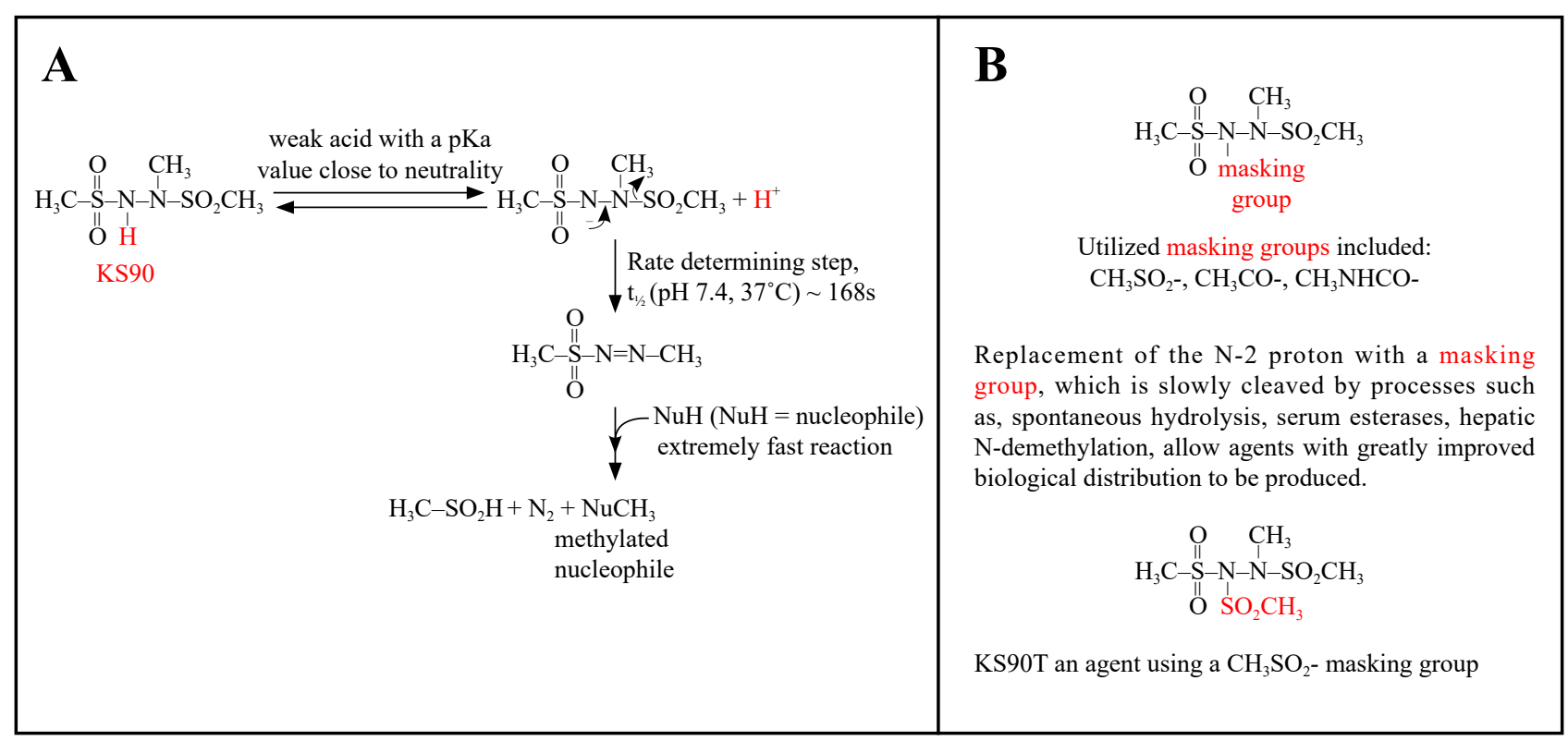

Figure 2. Methylating sulfonylhydrazines: (A) The methylation of biological nucleophiles by KS90; and (B) The production of longer lived prodrug forms by the addition of masking groups. 
The cures produced by repetitive low doses were due to the drug treatment inducing the entire population to differentiate into short stumpy, fly-infective forms that were incapable of division in the mammalian host and not simply due to a loss of viability since they continued their development to procyclic forms and could be maintained in culture if they were placed in Cunningham's medium (a mimic of the Tsetse fly gut). Thus, in this manner, they were identical to naturally occurring short stumpy forms. This drug-facilitated transition could be efficiently accomplished even with highly monomorphic strains which fail to make this conversion when transferred to Cunningham's medium [3]. Thus, highly monomorphic lines could be transformed from one extreme (all long slender) to the other (all short stumpy). In common with normal short stumpy forms, their drug-induced equivalents also exhibited $\alpha$-ketoglutarate supported motility and oxygen consumption, nicotinamide adenine dinucleotide (NADH) diaphorase positivity, and appeared to be in the $\mathrm{G}_{1}$ or $\mathrm{G}_{0}$ stage of the cell cycle [3]. Several ethylating and chloroethylating agents failed to elicit any significant response, but a wide range of methylating agents, ranging from soft agents, such as dimethyl sulfate, which favor the alkylation of soft nucleophilic sites [7, 8] to the much harder agents, N-methyl-N-nitrosourea [8] and KS90 [9], which favor the alkylation of hard nucleophilic sites were active [3]. This would imply that if these agents were all active via the methylation of the same target, this target would likely be one of intermediate hardness/softness, such as a nitrogenbased nucleophile, where there would be overlap in the methylating activity of these agents. The specific requirement for methylation over other alkyl groups tempts the speculation that these agents may be mimicking a natural biological trigger in these organisms. If the transition to short stumpy forms merely represented a general response of the long slender forms to electrophilic stress, one would expect to observe a similar effect by ethylating and chloroethylating agents, but none was observed [3, 6]. Difluoromethylornithine (DFMO) also induces differentiation in T. b. brucei albeit over a more protracted period [10]. This agent causes a 1,000-fold increase in decarboxylated $S$-adenosylmethionine (DSAM) and $S$-adenosylmethionine (SAM) within these trypanosomes. These latter metabolites are weak chemical methylating agents [11]. Thus, methylation could also play a role in DFMO-induced long slender to short stumpy form differentiation. SAM is also the methyl donor used by many methylases; therefore, enzymatically mediated methylation reactions may also be affected indirectly by DFMO. There has been interest in the epigenetic regulation of trypanosome differentiation via histone-modifying lysine methylases and demethylases [12]. Therefore, it is not implausible that natural regulatory switches could be activated by chemical methylating agents in trypanosomes.

Related compounds which donate a chloroethyl rather than a methyl group were potent antileukemia agents in model systems; some of the newer antileukemia agents owed their improved efficacy to superior pharmacokinetics [13]. It is therefore likely that methylating analogues of these newer agents (Figure 2) would similarly display superior efficacy against $T$. brucei.

Long slender bloodstream forms have a strong proclivity for the uptake of amidines, a consequence of the very promiscuous nature of their P2 aminopurine transporter [14]. Therefore, we synthesized amidine-containing methylators, with a methyl- or a dimethyltriazene moiety as the alkylating arm. The addition of the amidine functionality to target the trypanosomes resulted in an approximately 10 -fold increase in potency over equivalent agents containing a methyl but lacking an amidine group [15]. A similar strategy could be implemented to target prodrugs of KS90. The purinelike structure of the clinically used and surprisingly non-toxic methylator temozolomide may also aid in its uptake by trypanosomes. Temozolomide is used to treat tumors of the central nervous system in part because of its ability to readily cross the BBB [16]. Therefore, temozolomide may potentially be of utility in late-stage sleeping sickness, where the trypanosomes have circumvented the BBB [17] and become refractory to many conventional trypanocides. Recently, there have been some sulfonylhydrazine alkylator designs that are anticipated to increase their selectivity towards tumors. It is anticipated that some of these agents will also exhibit enhanced activity as trypanocidal agents [18]. A recent review of approved drugs with activity against African trypanosomiasis reveals that there have been very few advancements in this area in recent years [19]. Thus, there is a dire need for the development of new agents.

The ability of methylating sulfonylhydrazines to induce differentiation in bloodstream trypomastigotes of $T$. brucei group trypanosomes may also give them utility as tools for studying differentiation and its control in African trypanosomes, and targeted prodrugs of methylating sulfonylhydrazines could have therapeutic potential in the treatment of this devastating disease [3]. 


\section{Acknowledgments}

This research was supported in part by the National Foundation for Cancer Research.

\section{Conflict of interest statement}

There is no conflict of interest for this study.

\section{References}

[1] World Health Organization. The world health report 2004: Changing history. Geneva: World Health Organization; 2004. Available from: https://apps.who.int/iris/handle/10665/42891 [Accessed 30th November 2021].

[2] Matthews KR. The developmental cell biology of Trypanosoma brucei. Journal of Cell Science. 2005; 118(2): 283290. Available from: doi:10.1242/jcs.01649.

[3] Penketh PG, Divo AA, Shyam K, Patton CL, Sartorelli AC. The effects of methylating agents on morphology, DNA content and mitochondrial function in Trypanosoma brucei spp. The Journal of Protozoology. 1991; 38(3): 172177. Available from: doi:10.1111/j.1550-7408.1991.tb04425.x.

[4] Breidbach T, Ngazoa E, Steverding D. Trypanosoma brucei: in vitro slender-to-stumpy differentiation of cultureadapted, monomorphic bloodstream forms. Experimental Parasitology. 2002; 101(4): 223-230. Available from: doi:10.1016/S0014-4894(02)00133-9.

[5] Shyam K, Penketh PG, Divo AA, Loomis RH, Patton CL, Sartorelli AC. Synthesis and evaluation of 1,2,2-tris(sulfonyl)hydrazines as antineoplastic and trypanocidal agents. Journal of Medicinal Chemistry. 1990; 33(8): 2259-2264. Available from: doi:10.1021/jm00170a033.

[6] Penketh PG, Shyam K, Divo AA, Patton CL, Sartorelli AC. Methylating agents as trypanocides. Journal of Medicinal Chemistry. 1990; 33(2): 730-732. Available from: doi:10.1021/jm00164a041.

[7] Hoffmann GR. Genetic effects of dimethyl sulfate, diethyl sulfate, and related compounds. Mutation Research. 1980; 75(1): 63-129. Available from: doi:10.1016/0165-1110(80)90028-7.

[8] Coles B. Effects of modifying structure on electrophilic reactions with biological nucleophiles. Drug Metabolism Reviews. 1984; 15(7): 1307-1334. Available from: doi:10.3109/03602538409029962.

[9] Penketh PG, Shyam K, Sartorelli AC. Studies on the mechanism of decomposition and structural factors affecting the aqueous stability of 1,2-bis(sulfonyl)-1-alkylhydrazines. Journal of Medicinal Chemistry. 1994; 37(18): 29122917. Available from: doi:10.1021/jm00044a012.

[10] Giffin BF, McCann PP, Bitonti AJ, Bacchi CJ. Polyamine depletion following exposure to DL- $\alpha-$ difluoromethylornithine both in vivo and in vitro initiates morphological alterations and mitochondrial activation in a monomorphic strain of Trypanosoma brucei brucei. The Journal of Protozool. 1986; 33(2): 238-243. Available from: doi:10.1111/j.1550-7408.1986.tb05599.x.

[11] Rydberg B, Lindahl T. Nonenzymatic methylation of DNA by the intracellular methyl group donor $S$-adenosylL-methionine is a potentially mutagenic reaction. The EMBO Journal. 1982; 1(2): 211-216. Available from: doi:10.1002/j.1460-2075.1982.tb01149.x.

[12] Figueiredo LM, Cross GAM, Janzen CJ. Epigenetic regulation in African trypanosomes: a new kid on the block. Nature Reviews Microbiology. 2009; 7: 504-513. Available from: doi:10.1038/nrmicro2149.

[13] Penketh PG, Finch RA, Sauro R, Baumann RP, Ratner ES, Shyam K. pH-dependent general base catalyzed activation rather than isocyanate liberation may explain the superior anticancer efficacy of laromustine compared to related 1,2-bis(methylsulfonyl)-1-(2-chloroethyl)hydrazine prodrugs. Chemical Biology \& Drug Design. 2018; 91(1): 62-74. Available from: doi:10.1111/cbdd.13057.

[14] Stewart ML, Boussard C, Brun R, Gilbert IH, Barrett MP. Interaction of monobenzamidine-linked trypanocides with the Trypanosoma brucei P2 aminopurine transporter. Antimicrobial Agents and Chemotherapy. 2005; 49(12): 5169-5171. Available from: doi:10.1128/AAC.49.12.5169-5171.2005.

[15] Buddhu S, Penketh PG, Divo AA, Shyam K, Patton CL, Sartorelli AC. Enhancement of the trypanocidal activity of 
methyltriazenes in an animal model by the addition of an amidino group. Acta Tropica. 1991; 49: 69-72.

[16] Agarwala SS, Kirkwood JM. Temozolomide, a novel alkylating agent with activity in the central nervous system, may improve the treatment of advanced metastatic melanoma. The Oncologist. 2000; 5: 144-151. Available from: doi: 10.1634/theoncologist.5-2-144.

[17] Masocha W, Rottenberg ME, Kristensson K. Migration of African trypanosomes across the blood-brain barrier. Physiology and Behavior. 2007; 92(1-2): 110-114. Available from: doi:10.1016/j.physbeh.2007.05.045.

[18] Penketh P, Shyam K. Design strategy and approach to increase the selectivity of 1,2-bis(sulfonyl)-1-alkylhydrazine warheads in tumor-targeted applications. Biomedical Journal of Scientific \& Technical Research. 2021; 34(3): 26781-26790. Available from: doi:10.26717/BJSTR.2021.34.005558.

[19] De Koning HP. The drugs of sleeping sickness: their mechanisms of action and resistance, and a brief history. Tropical Medicine and Infectious Disease. 2020; 5(1): 14. Available from: doi:10.3390/tropicalmed5010014. 